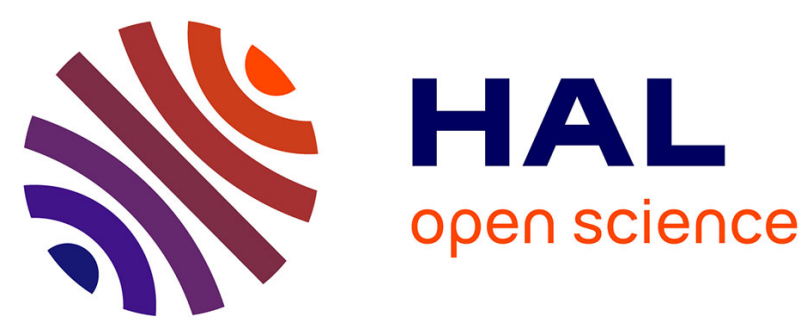

\title{
Autoantibodies against heat shock proteins as biomarkers for the diagnosis and prognosis of cancer
}

Liu Shi, Yann Chevolot, Eliane Souteyrand, Emmanuelle Laurenceau

\section{To cite this version:}

Liu Shi, Yann Chevolot, Eliane Souteyrand, Emmanuelle Laurenceau. Autoantibodies against heat shock proteins as biomarkers for the diagnosis and prognosis of cancer. Cancer Biomarkers, 2017, 18 (2), pp.105-116. 10.3233/CBM-160117 . hal-01701514

\section{HAL Id: hal-01701514 https://hal.science/hal-01701514}

Submitted on 26 Apr 2019

HAL is a multi-disciplinary open access archive for the deposit and dissemination of scientific research documents, whether they are published or not. The documents may come from teaching and research institutions in France or abroad, or from public or private research centers.
L'archive ouverte pluridisciplinaire HAL, est destinée au dépôt et à la diffusion de documents scientifiques de niveau recherche, publiés ou non, émanant des établissements d'enseignement et de recherche français ou étrangers, des laboratoires publics ou privés.

$$
\text { Copyright }
$$




\title{
Autoantibodies against heat shock proteins as biomarkers for the diagnosis and prognosis of cancer
}

\author{
Liu Shi ${ }^{1}$, Yann Chevolot ${ }^{1}$, Eliane Souteyrand ${ }^{1}$ and Emmanuelle Laurenceau ${ }^{1, *}$ \\ ${ }^{1}$ Université de Lyon; Institut des Nanotechnologies de Lyon INL-UMR5270, CNRS, Ecole Centrale de Lyon, \\ Ecully, F-69134, France \\ * Corresponding author \\ Email: emmanuelle.laurenceau@ec-lyon.fr \\ Tel : $+33(0) 472186240$ \\ Fax : +33(0)4 78331577
}

\begin{abstract}
Thanks to their specificity and stability in the sera, autoantibodies (AAbs) against tumor-associated antigens (TAAs) are very attractive biomarkers for the development of less invasive serological tests for the diagnosis and prognosis of cancer. Heat shock proteins (HSP) belong to TAAs and they are over-expressed in various human cancers. Elevated HSP can stimulate the immune system to produce anti-HSP antibodies. So far, AAbs against HSP have been identified in the circulation of various cancer patients. Here we will review current literature on the use of anti-HSP antibodies for cancer diagnosis and prognosis. The challenges as well as future directions of AAbs identification in oncology are also discussed.
\end{abstract}

Key words: heat shock proteins, autoantibodies, cancer, diagnosis, prognosis

\section{Introduction}

Cancer remains a major public health problem in the world. According to World Health Organization, in 2012 there were 14.1 million new cancer cases, 8.2 million cancer deaths and 32.6 million people living with cancer (within 5 years of diagnosis) worldwide [1]. Early detection represents one of the most promising approaches to reduce the growing cancer burden [2]. One of conventional diagnostic method is clinical examination, which is widely used for breast cancer diagnosis. However, it is not recommended because women will be over-diagnosed and receive needless treatment [3]. Another method is image analysis, such as, mammography and magnetic resonance imaging for breast cancer, colonoscopy for colorectal cancer, endoscopic examination for esophageal squamous cell carcinoma (ESCC), etc. However, the use of these procedures is limited by various factors. Mammography and MRI for breast cancer diagnosis were questioned by their harms of causing false positive and unnecessary biopsies [4]. Colonoscopy and endoscopic examination are too invasive for screening colorectal cancer and ESCC respectively [5, 6]. Furthermore, malignant cancers which are associated with higher mortality frequently cannot be detected by routine screening [7].

Recent research has demonstrated that analyzing biomarkers in sera could be a less-invasive and practical approach for cancer diagnosis. According to Etzioni et al, effective screening tests should satisfy four basic requirements: 1) Screening tests should be able to distinguish healthy individuals from cancer cases with a high degree of accuracy, showing both low false negative and low false positive rates. 2) Detection should be possible before the disease progresses to an advanced stage, when treatment is less effective. 3) Screening or formal diagnostic tests should ideally allow differentiation between aggressive lesions that require treatment and those that ultimately will do no harm, avoiding the problem of over-diagnosis. 4) Tests should be inexpensive and well accepted by the population targeted for screening [2]. These criteria preclude many currently used markers. For example, CA-15-3 glycoprotein is used as biomarker for the detection of breast carcinoma. However, the concentration of CA 15-3 antigen increased in 10\% of patients with stage I disease, $20 \%$ with stage II disease, $40 \%$ with stage III disease and $75 \%$ with stage IV disease. Therefore, lack of sensitivity for early-stage disease combined with a lack of specificity precludes the use of CA 15-3 antigen for the early diagnosis of breast cancer [8]. CA-125 glycoprotein, a biomarker for the detection of epithelial ovarian carcinoma, has also been limited by its insufficient specificity and sensitivity, particularly for organ confined early-stage disease. Elevated levels of CA-125 occur in only 50\% of stage I patients and can also be detected in healthy women [9]. CYFRA21-1 and squamous cell carcinoma antigen are currently used in clinical practice for early-stage squamous cell carcinoma (ESCC) diagnosis. While the positive frequencies of CYFRA21-1 are reported to be only $4.7 \%$ and $25 \%$ in stage I and stage II cancers, respectively. For squamous cell carcinoma antigen, positive frequencies in patients with stage $0+$ I and stage II have been reported to be $10.8 \%$ and $24.0 \%$, respectively [10]. Prostate-specific antigen (PSA) was used to diagnose prostate cancer and the assay is well standardized. PSA is almost exclusively produced by prostate tissue and elevated level can be detected 5-10 years prior to a clinical diagnosis 
of prostate cancer. However, PSA lacks sensitivity and specificity. At the commonly used cut-off point of 4 $\mu \mathrm{g} / \mathrm{L}$, the sensitivity of PSA is only approximately $20 \%$ and specificity only $60-70 \%$. Therefore, screening test of PSA could result to unnecessary biopsies and over treatments [11]. In a word, all these biomarkers have limited clinical use for diagnosis due to their insufficient specificity and sensitivity.

Over the past few years, an increasing number of researches have shown that human tumors stimulate the immune system to produce autoantibodies (AAbs) against autologous cellular proteins called tumor-associated antigens (TAAs). AAbs are often driven by intracellular proteins that are mutated, modified, or aberrantly expressed in tumor cells. AAbs have several advantages over other serum proteins as potential cancer biomarkers. They are stable, highly specific, easily purified from serum, and are readily detected with wellvalidated secondary reagents. Furthermore, this immune response seems to appear months to years before the clinical diagnosis of a tumor, rendering serum AAbs detection suitable for early-stage cancer diagnosis [12-14]. Because of the heterogeneity of tumor, emerging evidence suggests that each type of cancer might trigger unique AAbs signatures that reflect the nature of the malignant process in the affected organ [15]. Among the numerous AAbs found in cancer patients, AAbs against heat shock proteins (HSP) were described in a wide range of cancers. After a brief introduction on the biological functions of HSP, this review provides a survey of results obtained using individual AAbs against HSP as well as multi-AAbs panels to discriminate cancers from healthy controls. We will also discuss the specific challenges concerning the development of AAb biomarkers and lay the groundwork for its future innovations.

\section{Heat shock proteins (HSP)}

HSP were first discovered as a cohort of proteins that are powerfully induced by heat shock and other chemical or physical stresses in a wide range of species [16]. HSP are a group of highly conserved proteins and are classified into six families according to their molecular weight (MW): HSP110, HSP90, HSP70, HSPD1, DNAJ and small HSPs (ranging between 13-42kDa) including HSPB1 and HSP10. Glucose-regulated proteins (GRP) are a related class of proteins which are localized in endoplasmic reticulum. For example, HSPA5 belongs to HSP70 family member and shares 60\% amino acid identity with HSP70; HSP90B1 belongs to HSP90 family and shares 50\% amino acid identity with HSP90 [17]. HSP function predominantly as molecular chaperones. They also restore cellular homeostasis by ensuring proper formation of new proteins, preserving existing complexes, restoring function of denatured proteins, and solubilizing protein aggregates [18].

HSP are over-expressed in a wide range of human cancers. Elevated HSP expression in malignant cells plays a key role in protecting cells against the spontaneous apoptosis associated with malignancy. Several HSP are associated with the prognosis of specific cancer. For example, the expression of HSPB1 is associated with poor prognosis in gastric, liver, and prostate carcinoma, and osteosarcomas; overexpression of HSP70 is correlated with poor prognosis in breast, endometrial, uterine cervical, and bladder carcinomas [19]. Increased HSP expression may also predict the response to some anti-cancer treatments. HSPB1 and HSP70 were shown to be involved in resistance to chemotherapy in breast cancer; HSPB1 predicted a poor response to chemotherapy in leukemia patients, whereas HSP70 expression predicted a better response to chemotherapy in osteosarcomas. Furthermore, implication of HSP in tumor progression and response to therapy has led to its successful targeting in therapy [19]. Thus, the detection of HSP as biomarkers of cancer could aid early diagnosis, determining prognosis, prospectively predicting response or resistance to specific therapies, surveillance after primary surgery, and monitoring therapy in patients with advanced disease [18, 19].

Elevated HSP expression in tumor can also stimulate the immune system to produce anti-HSP AAbs. Conroy SE et al. [20] found that AAbs against HSP90 were detectable in a significant proportion (37\%) of patients with breast cancer but not in normal individuals or patients with benign breast tumors. AAbs against HSP are also related with prognosis. Same authors [21] demonstrated that mortality rate from breast carcinoma was greater in women tested positive for AAbs against HSP90 than those tested negative. Thus, AAbs against HSP could also have diagnostic and prognostic values in cancer. Furthermore, AAbs have a number of advantages over traditional protein biomarkers. Unlike the short-lived changes of tumor-antigens in serum, the antibody molecule is stable in the blood and antibody response is enduring. Moreover, the nature of antibody amplification response to antigen means that even relatively small quantity of antigen can stimulate larger immune response, leading to higher concentration of antibody [13]. In the following, we reviewed literature about AAbs against HSP family in eight most common cancers including lung cancer, breast cancer, colorectal cancer, gastric cancer, prostate cancer, liver cancer, cervix cancer and esophagus cancer. These eight cancers accounted for more than $62 \%$ of all cancers' incidence in 2008 according to WHO-International Agency for Research on Cancer [1]. 


\section{Anti-HSP AAbs in cancer}

\section{Evaluation of single anti-HSP autoantibodies (AAbs) in various cancers}

17 reports described the use of single anti-HSP AAb for discriminating cancer patients from healthy controls. A summary of these studies is presented in table 1. Anti-HSP autoantibodies were studied in breast cancer, ovarian cancer, hepatocellular carcinoma (HCC), esophageal squamous cell carcinoma (ESCC), colorectal cancer (CRC), gastric cancer, prostate cancer and non-small cell lung cancer (NSCLC). Among all these studies, 13 used ELISA and 4 used immunoblot for the detection of anti-HSP autoantibodies. From these data we can observe that the frequency of a single anti-HSP autoantibody in cancer patients ranged from $8 \%-40 \%$ (some extremely high or low frequency was not included), whereas the frequency in healthy controls ranged from $1.6 \%-25 \%$.

Autoantibodies against HSP were found be useful to discriminate breast cancer patients from healthy controls. Using home-made ELISA, in 1995, Conroy et al. conducted the first study to identify anti-HSP90 autoantibodies in patients diagnosed with breast cancer. They found that autoantibodies targeting purified HSP90 were detectable in $46 / 125(36.8 \%)$ breast carcinoma patients but not in healthy individuals, or patients with benign breast tumors. Furthermore, the presence of these autoantibodies was found to be correlated with the development of metastasis even in patients without axillary nodal involvement [20]. Then, in another study, they analyzed the correlation between anti-HSP90 AAbs and mortality rate. They found that mortality rate from breast carcinoma was greater in women tested positive for AAbs against HSP90 than those tested negative [21]. This research group also identified autoantibodies against HSPB1 and HSP70 in breast cancer, still using homemade ELISA. One of the largest sample cohort was evaluated with 579 samples tested for anti-HSPB1 autoantibodies and 369 samples tested for anti-HSP70 autoantibodies. In comparison, the number of healthy controls (53 healthy female) were limited. Results showed that there was no significant difference in the frequency of anti-HSP70 autoantibodies in patients with breast cancer and healthy control subjects. In contrast, anti-HSPB 1 autoantibodies were detectable in over one-third of breast cancer patients (37.8\%) while only in one healthy individual $(\mathrm{P}<0.001)$. Furthermore, the presence of anti-HSPB1 autoantibodies appeared to show a significant correlation with improved survival, particularly beyond the first 5 years [22]. In contrast, no antiHSPB1 autoantibodies were found in serum from both breast cancer patients and healthy controls by immunoblotting according to Fanelli MA et al. Therefore, they concluded that serological determination of this biomarker is unlikely to be of utility in the detection of breast cancer patients [23]. However, it should be noticed that limited sample size was involved (11 breast cancer patients and 5 healthy controls). Two studies were conducted concerning to the frequency of anti-HSPD1 autoantibodies in breast cancers. Hamrita et al. used immunoblot analysis on a cohort of 40 patients with invasive breast cancer and 42 healthy controls. A significantly higher frequency of anti-HSPD1 autoantibodies was observed in breast cancer patients group $(19 / 40,47.5 \%)$, compared to control sera group $(2 / 42,4.7 \%)$. Thus, they suggested that circulating anti-HSPD1 autoantibodies could display clinical usefulness as diagnostic markers for breast cancer [24]. This was confirmed by Desmetz et al. in a study included 49 ductal carcinoma in situ (DCIS) patients, 58 early stage breast cancer patients, 93 healthy controls, 20 other cancer patients and 20 autoimmune diseases [25]. They used commercial ELISA kit and found that anti-HSPD1 autoantibodies were detected in $32.6 \%(16 / 49)$ patients with DCIS and $31 \%$ (18/58) patients with early stage breast cancer, compared with $4.3 \%$ (4/93) in healthy controls and $0 \%$ in other control groups. Furthermore, the presence of anti-HSPD1 autoantibodies was closely associated with disease grade in DCIS. Indeed, Anti-HSPD1 autoantibodies were found in 11/23 patients (47.8\%) with highgrade DCIS, compared with $5 / 26$ patients $(19.2 \%)$ with low-grade DCIS ( $\mathrm{p}=0.0188)$. Anti-HSPD1 autoantibodies displayed a specificity of $95.7 \%$ and a sensitivity of $31.8 \%$ for discriminating breast cancer patients from healthy controls. Furthermore, the area under the curve (AUC) obtained from the receiver operating characteristics curves (ROC) is $63.7 \%$.

In ovarian cancer, conflicting results about the usefulness of anti-HSP autoantibodies were reported. Using home-made ELISA, Irina Korneeva et al. identified autoantibodies against HSPB1, HSPD1, HSP70 and HSP90 in patients diagnosed with ovarian cancer. Among all autoantibodies studied, anti-HSPB1 autoantibodies were detected in 50\% (17/34) of ovarian cancer patients, while only 1 of 29 healthy control subjects (3.4\%) and 1 of 23 women whose lesions were benign (4.3\%). In contrast, autoantibodies against HSPD1, HSP70 and HSP90 could not discriminate ovarian cancer patients from healthy controls or benign disease, as shown is table 1 [27]. Opposite results were obtained from Luo et al. [26], indicating that anti-HSP90 autoantibodies were significantly detected in $25 \%(8 / 32)$ of ovarian cancer patients, compared with benign gynecologic diseases (5\%) and healthy controls $(0 \%)$. Furthermore, anti-HSP90 AAbs were more frequently detected in late stage ovarian cancer (32\% of patients with stage III/IV ovarian carcinoma). So the authors proposed that anti-HSP90 autoantibodies could be used as a novel prognostic biomarker for ovarian cancer. Furthermore, in this study, other types of cancer were screened for the presence of anti-HSP90 autoantibodies and results showed that they could not discriminate 
healthy donors from breast cancer, colorectal cancer, lung cancer and prostate cancer patients. However, it should be noted that the samples size of this study was relatively small. The discrepancy between these 2 studies could originate from the origin of antigens. Both two studies used home-made ELISA and they purchased commercial purified HSP90 from StressGen Biotechnologies Corp. However, in the study of I. Korneeva et al. [27], they used recombinant proteins while in the one of L. Y. Luo et al. [26], they used purified HSP90 isolated from HeLa cells. These two kinds of proteins could have different conformation or post-translational modifications; therefore, they could have different reaction with autoantibodies exist in human serum, thus leading to the discrepancy of results. In another approach, HSP90-mimic peptide was developed for ELISA to detect anti-HSP90 autoantibodies in ascites from patients with advanced ovarian cancer (stages III and IV), metastatic gastrointestinal cancer, and nonmalignant cirrhosis [28]. For ovarian cancer patients, the level of antiHSP90 autoantibodies in ascites was found to be closely associated with disease stage (3/42 patients with stage III disease versus 10/17 patients with stage IV disease). Anti-HSP90 autoantibodies were also detected in gastrointestinal cancer ascites $(13.3 \%)$ but not in cirrhotic ascites $(0 \%)$. The authors concluded that the humoral response against HSP90 in ovarian cancer is stage-specific and anti-HSP90 autoantibodies could have prognostic value in malignant tumors. However, this conclusion should be nuanced because ascites samples are not common in clinic diagnosis. Moreover, HSP90-mimic peptide used targeted only one population of anti-HSP90 autoantibodies and its specificity to ovarian cancer should be confirmed with larger cohort samples.

Three studies focused on autoantibodies against individual HSP in hepatocellular carcinoma cancer (HCC). In 2006, Motonari Takashima et al. detected the presence of anti-HSP70 autoantibodies using immunoblot and found significant difference between HCC patients' sera (46.7\%) and healthy individuals (10\%). Thus, the authors concluded that AAb against HSP70 could be candidate diagnostic biomarkers for HCC [31]. However, the sample size of this study was very small (15 HCC sera and 20 healthy sera). In another study, 28 HCCrelated TAAs were firstly screened by two-dimensional gel electrophoresis (2-DE) and liquid chromatographytandem mass spectrometry (LC-MS/MS) in HCC sera and in pre-HCC sera like chronic hepatitis $(\mathrm{CH})$ and liver cirrhosis (LC). 17 TAA, including HSPD1, were reactive in all sera and 11 TAA, including HSP70, were only reactive in HCC sera. Thus, the presence of autoantibodies against HSPD1 and HSP70 were evaluated using home-made ELISA in sera from 20 patients with HCC, 30 patients with $\mathrm{CH}, 30$ patients with LC and in sera from 10 normal individuals. Anti-HSP70 autoantibodies were significantly detected in 25\% (5/20) HCC sera compared to LC sera (3.3\%) or $\mathrm{CH}$ and normal controls (0\%) suggesting that anti-HSP70 AAbs could be used as marker in HCC. In contrast, anti-HSPD1 autoantibodies were not able to discriminate HCC sera (20\%) from LC sera $(36.7 \%)$ and $\mathrm{CH}$ sera $(26.7 \%)$. Therefore, the authors concluded that the detection of autoantibodies against TAAs in pre-cancer conditions might not be good criteria for selection as markers for cancer detection [29]. However, chronic liver diseases such as chronic hepatitis and liver cirrhosis are clinically considered as early stages of HCC and patients with chronic liver diseases are most likely to develop HCC after more than ten years. Thus, more information about the evolution of patients' disease would be necessary to conclude on the real potential to use anti-HSPD1 autoantibodies as HCC biomarker. At last, the frequency and titer of anti-HSPA5 autoantibodies were found to be significantly higher in HCC sera $(35.5 \%)$ than in $\mathrm{LC}, \mathrm{CH}$ and normal human sera $(0 \%)$ suggesting their potential use as diagnostic biomarker for HCC [30]. Autoantibodies against HSP70 and HSPA5 also displayed high frequency in esophageal squamous cell carcinoma (ESCC) compared to gastric cancer patients, colon cancer patients and healthy individuals [32, 33].

Serological proteomics analysis (SERPA) on few serum samples (25 patients with CRC and 15 healthy subjects) combined with home-made ELISA screening of larger cohort (60 CRC patients, 20 breast cancer patients, 20 gastric cancer patients and 30 healthy individuals) allowed the identification of anti-HSPD1 autoantibodies as potential novel biomarker for CRC diagnosis [35]. However, the authors did not provide any information on the frequency of autoantibody against HSPD1 in CRC. Furthermore, conflicting results were observed concerning anti-HSPD1 autoantibodies in breast cancer. In this study, no significant difference was observed concerning serum autoantibody levels to HSPD1 in patients with breast cancer and healthy subjects. However, results obtained by Hamrita et al. [24] and Desmetz et al [25] found that anti-HSPD1 autoantibodies display high clinical usefulness as diagnostic markers for breast cancer. These two studies enrolled larger samples and indicated that sample size is a key factor in biomarker screening.

Anti-HSPA5 autoantibodies were also identified in gastric cancer using immunoblot analysis. However, they were shown to be also present in other studied cancers such as esophageal cancer and colon cancer [34]. Thus, it is difficult to conclude that anti-HSPA5 autoantibodies were specific and sensitive enough to be used as biomarker for the detection of gastric cancer. In prostate cancer, a peptide motif related to HSPA5 (sequence CNVSDKSC) was shown to significantly react with autoantibodies from prostate cancer sera (37\%) and weakly with healthy individuals' sera (7\%). Moreover, serum positivity to this peptide was closely correlated with the natural progression of the disease. Among all patients, only $6 \%$ of patients with organ-confined prostate cancer 
reacted with the peptide, whereas $29 \%$ of patients with metastatic androgen dependent tumors and $76 \%$ of patients with metastatic androgen independent prostate cancers displayed reactivity against the peptide [36].

At last, the presence of autoantibodies against HSP70 and HSP90 was studied in sera from 49 non-small cell lung cancer (NSCLC) patients and 40 healthy controls. Anti-HSP70 antibodies were significantly detected in NSCLC sera whereas anti-HSP90 antibodies were not. The AUC for anti-HSP70 autoantibodies was calculated to be 0.731 with an optimal predictive accuracy of sensitivity of 0.74 and a specificity of 0.73 . Thus, authors concluded that anti-HSP70 autoantibodies could be a modest biomarkers for lung cancer detection [37].

As surveyed above, the frequency of single anti-HSP autoantibodies in cancer patients range from $8 \%-40 \%$. This is maybe caused by internal (the heterogeneity of tumor) as well as external factors (discrepancy between studies). External factors include: 1) sample size: big range of sample size (from 10 to 579) were observed across these studies. As each person might trigger unique AAbs signatures because of the heterogeneity of tumor; therefore, the enrollment of sample size could have an influence on the frequency of aotuantibodies against HSPs in cancer. 2) Antigens resources are different. Only one study used commercial ELISA, others used home-made ELISA or Immunoblot with commercial HSPs. However, these commercial HSPs were provided by different companies, among which several of them are recombinant purified HSPs, while others are isolated from cell lines. HSPs with different origin could have different reaction with autoantibodies exist in human serum, thus leading to the discrepancy of results. 3) Cut-off was diversely defined. For example, in the study of I. Korneeva et al. [27] and L. Y. Luo et al. [26], they defined cut-off value as mean of healthy controls plus 2SD (SD represents standard deviation); while in the one of Q. Shao et al. [30], they defined it as mean of healthy controls plus 3SD. Generally, lower cut-off value result in higher sensitivity and lower specificity, and vice versa. Thus data analysis and definition of cut-off values for identification of positive or negative samples may result in the discrepancy of the frequency obtained. Furthermore, it is difficult to compare results across studies if different cut-off value was used. Compared with cutoff value, AUC is more standardized, comprehensive and widely used for measuring a diagnostic test's discriminatoAUC

ry power. In general, ROC curves with an AUC $\leq 0.75$ are not clinically useful and an AUC of $\geq 0.97$ has a very high clinical value [38]. Among 17 reports summarized above, only 2 of them analyzed AUC data. The AUC of anti-HSPD1 autoantibodies in breast cancer is 0.637 [25] and the AUC of anti-HSP70 autoantibodies in NSCLC is 0.731 [37]. Therefore, the use of single AAb as diagnostic biomarker remains limited due to the heterogeneity of tumor. Over the past 10 years several researches demonstrated that AAbs panels could greatly improve cancer sensitivity detection while preserving reasonable high level of specificity.

\section{Evaluation of AAbs panels including anti-HSP autoantibodies}

Thanks to the novel emerging proteomic techniques, like phage display, serologic identification of antigens by recombinant expression (SEREX) and serological proteome analysis (SERPA), various biomarkers which could discriminate cancers from healthy controls were discovered. In the following, we presented data obtained from AAbs panels (between 2 to 14 AAbs) including one or more anti-HSP autoantibodies (as shown in table 2). AAbs panels were studied in various cancers mostly using ELISA; high throughput screening tools such as protein microarrays were also used.

As could be seen on table 2, the number and the choice of autoantibodies used in each panel varied between studies on the same type of cancer and on different type of cancer. For breast cancer, 2 panels of 5 autoantibodies, including only anti-HSPD1 autoantibody as common antibody, were evaluated [39, 40]. In the study of C. Desmetz et al. [39], they chose the panel according to their proteomic approaches showed as follow: firstly, proteins from cancer cell lines were separated by 2-DE, and then followed by western blotting and antibody reaction with sera from cancer and healthy controls. Finally, positive spots were excised from Coomassie blue stained gels and analyzed by matrix-assisted laser desorption/ionization time of-flight mass spectrometry (MALDI-TOF/TOF MS). While in the study of Z. Yang et al. [40], they chose the antibody panel according to the reports in literature. Furthermore, in the study of C. Desmetz et al. [39], they involved more sample size and analyzed the AUC of the antibodies panel. Moreover, they have shown that their combination of 5 autoantibodies could discriminate CIS from healthy controls in women under the age of 50 years $(\mathrm{AUC}=0.85$; 95\% CI, 0.61-0.92) [39]. This result is very important for young women with high risk of developing invasive and aggressive tumors.

Profiling of autoantibodies in lung cancer received one of the highest attentions among all cancers. All panels identified exhibited good results in terms of sensitivity and specificity for the detection of NSCLC. The panels consisted of 2 to 9 autoantibodies in which anti-HSP70 autoantibody is the only common autoantibody. By adding 3 more autoantibodies in their panel, Zhong et al. could increase the sensitivity of NSCLC detection from 
$78 \%$ [37] to $82 \%$ [44] and the specificity from $65 \%$ to $83 \%$. Furthermore, AUC increased from 0.74 to 0.84 . However, only 16 patients were enrolled in this second study [44], which could cause overfitting bias. Overfitting occurs when a multi-markers panel is inappropriately large with respect to the number of cases evaluated. The prediction will thus be out of range because of noise. Generally, when the ratio of cases to markers is less than 10, it is considered of potential bias [48]. In this study, the ratio of cases to markers was 3.2 (16 lung cancer patients and 5 biomarkers), so the high level of discrimination may be caused by overfitting bias. They also assessed lung cancer risk in human populations by screening a panel of 9 autoantibodies (including the previous 5 autoantibodies) in 100 atypical adenomatous hyperplasia (AAH) subjects and 200 healthy controls with protein microarray. AAH is known to be associated with the development of malignant lesions in the lung, which belong to the pre-neoplastic lung lesions. Using this combination of 9 autoantibodies, the sensitivity and specificity could reach $82 \%$ and $70 \%$, respectively. So detecting a combination of AAbs could provide the information for assessing lung cancer risk and could be used for early diagnosis or screening of lung cancer [46]. At last, using immunobead assay and a combination of 6 other autoantibodies including anti-HSP70 autoantibody, NSCLC patients were efficiently discriminated from healthy controls with an observed AUC of 0.934, which have a high potential utility as a diagnostic test [45].

During the last decade, 3 studies reported the screening of autoantibody panels including anti-HSP autoantibody in HCC. The smallest panel consisted of 2 autoantibodies including anti-HSPA5 autoantibody, and allowed the identification of $71.4 \%$ of HCC patients using ELISA [30]. In the other two studies [41, 42], the panel of autoantibodies was larger ( 7 and 10 autoantibodies) and was selected after screening of more than 20 autoantibodies towards hundreds HCC patients and healthy controls. Both studies selected anti-HSPA5 autoantibody in their panel. In one study, with a panel of 7 autoantibodies selected from 26 phage-expressed antibodies (including antibody against HSPA5 and HSP70), HCC patients could be significantly discriminated from controls (chronic hepatitis patients and normal individuals) with AUC equal to 0.917, which have a high potential utility [41]. From another panel of 21 autoantibodies, identified by screening 96 patients with confirmed HCC and 96 healthy controls, 10 autoantibodies gave reproducible results with $91 \%$ specificity and $41 \%$ sensitivity [42]. This high specificity and low sensitivity maybe caused by the cutoff value. They define it as mean of healthy control plus $4 \mathrm{SD}$, which is higher than generally used (mean $+3 \mathrm{SD}$ ). As cutoff was differently defined across studies and they didn't provide AUC, therefore, it is difficult to compare the performance with others. However, two aspects of this study are encouraging and should be noted. Firstly, they checked the reproducibility of their autoantibody panels and avoided overfitting bias (inappropriate large panel of markers). Secondly, healthy control sera were well matched on age, gender as well as smoking history with HCC patients. These parameters are crucial for the development of reliable diagnosis assays.

Autoantibody profiling was also studied in some other cancers, such as prostate cancer [43], esophageal squamous cell carcinoma (ESCC) [10] and cholangiocarcinoma (CCA) [47]. Only the study on prostate cancer used commercial protein microarray (nitrocellulose coated FAST slides) to screen a panel of 14 AAbs including anti-HSP110 autoantibody. Although prostate cancer patients could be discriminated from patients with benign disease (elevated PSA levels but prostate cancer negative biopsies) (AUC of 0.71) and from healthy controls (AUC of 0.69), this panel was not sensitive and specific enough for use in clinical practice. Moreover, this study probably had overfitting bias since 14 biomarkers were studied for only 40 cancer patients. In ESCC, a panel of 6 AAbs, including anti-HSP70 autoantibody, was assessed using ELISA on a test cohort (composed of 388 patients with ESCC and 125 normal individuals) and an independent validation cohort (composed of 237 patients and 134 normal controls). Similar results were obtained from the two cohorts. The tested panel could discriminate early-stage ESCC patients from normal controls with around 45\% sensitivity and $95 \%$ specificity. No significant difference was obtained between early stage patients and late stage patients. However higher incidence of AAbs was observed in patients with late stage tumors than in early stage groups, suggesting that AAbs could be associated with development of tumor metastasis. At last, a panel of only 3 AAbs, including antiHSP70 autoantibody, was screened in 31 CCA and 23 healthy controls. Among all three AAbs tested, antiHSP70 autoantibody was significantly capable to discriminate CCA from healthy individuals with AUC of 0.9158. Furthermore, this antibody was correlated with progression from healthy individuals to cholangitis to CCA. However, this study is limited by sample size.

In all these studies, the presence of several anti-HSP autoantibodies was evaluated (autoantibodies against HSPD1, HSP70, HSP90, HSP110 and HSPA5) alone or in combination with other AAbs for the diagnosis of various cancers. For most of them, patients' cohorts used were larger than the ones used for single anti-HSP antibody analysis providing more reliable statistical analysis. However, up to date, there was no comparative analysis on these data to determine the diagnostic potential of anti-HSP AAbs detection in sera. 


\section{Emerging trends of anti-HSP AAbs detection in cancer diagnosis}

We compiled and compared data on anti-HSP AAbs frequency obtained from the various studies described above. Results are presented in table 3. The most commonly studied antibody was anti-HSP70 autoantibody, which was reported in 9 separate investigations concerning 7 different cancers. Autoantibodies against HSPD1, HSPA5 and HSP90 also received much interest. However, autoantibodies against HSPB1 and HSP110 were poorly studied.

Considering a given anti-HSP autoantibody, its frequency varied with the type of cancer and with the size of the sample studied. For example, the frequency of anti-HSP70 autoantibody in breast, ovarian, HCC, ESCC, CRC, gastric and NSLC varied from $8 \%$ to $93.7 \%$ (Table 3). But if we consider the frequency of anti-HSP70 autoantibody in ESCC, study on few samples (16 cases) led to high frequency (93.7\%) [32]. However, increasing sample size to 69 cases and 237 cases, the frequency of anti-HSP70 autoantibody decreased from $93.7 \%$ to $39.1 \%$ and $8 \%$, respectively $[10,33]$. Concerning anti-HSP90 autoantibody, its frequency was also dependent on the type of cancer (NSCLC, Prostate, CRC, HCC, breast, ovarian) with variation from 0 to $25 \%$. However, in the case of breast cancer, larger samples led to higher frequency [20,26]. From this analysis, it is clear that sample size, sample quality (including both cases and well matched healthy controls) and sample origin are important parameters in the identification and determination of accurate anti-HSP autoantibodies profile in cancer. Secondly, with comparable samples, the sensitivity of the method used for the detection of anti-HSP autoantibodies is also very important. Indeed, in the case of HCC (15-20 cases), the frequency of antiHSP70 autoantibody was $46.7 \%$ with immunoblot analysis [31] versus $25 \%$ with ELISA [29]. It is well-known that immuno-blot is more sensitive method than ELISA [49]. However, ELISA is routinely used in clinical diagnosis because of commercially available kit and automation, while immuno-blot is time-consuming and laborious. Both methods are not easily implemented for multiplex analysis and consume large amounts of biological products and patient specimen, which is obviously not suitable for large-scale investigations. The need for efficient large scale investigation has stimulated the development of novel technologies, e.g. protein microarray, which could provide multiple and parallel protein measurements simultaneously by consuming minute samples [50].

\section{Conclusions}

An increasing number of researches have found that identifying autoantibodies (AAbs) against HSP can aid early diagnosis of cancer. Furthermore, since autoantibodies against HSP are associated with tumor stage, identifying them might monitor disease progression and provide prognostic implications. However, since the origin and mechanism of immune response involved in cancer is not yet fully understood, more basic studies concerning elucidating the mechanism of anti-HSP antibodies involved in tumorigenesis are need to accelerate its clinic use. Furthermore, due to the heterogeneity of tumor, the detection of individual AAb for diagnosis purpose has been tempered by their low frequency (range from 8\%-40\%). The detection of a panel of AAbs has shown to greatly improve the discrimination between cancer patients and healthy controls. Two aspects need to be noted for further study. Firstly, data analysis should be standardized and comprehensive. As mentioned in the text, AUC analysis is better than defining cutoff as a certain value. Only when we standardized data analysis method, could we compare the results across studies. Secondly, large systematic and unbiased prospective studies is urgently needed in order to validate the true diagnostic value of each AAb or a panel of AAbs. Although challenges exist, identification of autoantibodies in cancers have great potential to be useful and informative biomarkers of cancer diagnosis and prognosis.

\section{Acknowledgements}

China Scholarship Council is grateful to offer Ph.D. fellowship to Liu SHI. The CNANO Rhone-Alpes and BQR Ecole Centrale de Lyon are acknowledged for financial support.

\section{Reference}

[1] M. T. Aloy, E. Hadchity, C. Bionda, C. Diaz-Latoud, L. Claude, R. Rousson, et al., Protective role of Hsp27 protein against gamma radiation-induced apoptosis and radiosensitization effects of Hsp27 gene silencing in different human tumor cells, Int J Radiat Oncol Biol Phys, 70 (2008), 543-553. 
[2] R. Etzioni, N. Urban, S. Ramsey, M. McIntosh, S. Schwartz, B. Reid, et al., The case for early detection, Nat Rev Cancer, 3 (2003), 243-252.

[3] M. S. Fuller, C. I. Lee, and J. G. Elmore, Breast Cancer Screening: An Evidence-Based Update, Med Clin North Am, 99 (2015), 451-468.

[4] J. Brodersen, K. J. Jorgensen, and P. C. Gotzsche, The benefits and harms of screening for cancer with a focus on breast screening, Pol Arch Med Wewn, 120 (2010), 89-94.

[5] C. S. Lee, L. Ronan, C. O'Morain, and D. McNamara, Screening for colorectal cancer: what fits best?, Expert Rev Gastroenterol Hepatol, 6 (2012), 301-312.

[6] G. Roshandel, A. Nourouzi, A. Pourshams, S. Semnani, S. Merat, and M. Khoshnia, Endoscopic screening for esophageal squamous cell carcinoma, Arch Iran Med, 16 (2013), 351-357.

[7] C. Desmetz, A. Mange, T. Maudelonde, and J. Solassol, Autoantibody signatures: progress and perspectives for early cancer detection, J Cell Mol Med, 15 (2011), 2013-2024.

[8] M. J. Duffy, Serum tumor markers in breast cancer: are they of clinical value?, Clin Chem, 52 (2006), 345-351.

[9] B. Piura and E. Piura, Autoantibodies to tumor-associated antigens in epithelial ovarian carcinoma, $J$ Oncol, 2009 (2009), 1-11.

[10] Y. W. Xu, Y. H. Peng, B. Chen, Z. Y. Wu, J. Y. Wu, J. H. Shen, et al., Autoantibodies as potential biomarkers for the early detection of esophageal squamous cell carcinoma, Am J Gastroenterol, 109 (2014), 36-45.

[11] M. J. Duffy, PSA in screening for prostate cancer: more good than harm or more harm than good?, $A d v$ Clin Chem, 66 (2014), 1-23.

[12] E. Piura and B. Piura, Autoantibodies to tumor-associated antigens in breast carcinoma, J Oncol, 2010 (2010) 1-14.

[13] C. K. Heo, Y. Y. Bahk, and E. W. Cho, Tumor-associated autoantibodies as diagnostic and prognostic biomarkers, BMB Rep, 45 (2012), 677-685.

[14] H. Lu, V. Goodell, and M. L. Disis, Humoral immunity directed against tumor-associated antigens as potential biomarkers for the early diagnosis of cancer, J Proteome Res, 7 (2008), 1388-1394.

[15] C. A. Casiano, M. Mediavilla-Varela, and E. M. Tan, Tumor-associated antigen arrays for the serological diagnosis of cancer, Mol Cell Proteomics, 5 (2006), 1745-1759.

[16] S. Lindquist and E. A. Craig, The heat-shock proteins, Annu Rev Genet, 22 (1988), 631-677.

[17] Q. Wang, Z. He, J. Zhang, Y. Wang, T. Wang, S. Tong, et al., Overexpression of endoplasmic reticulum molecular chaperone GRP94 and GRP78 in human lung cancer tissues and its significance, Cancer Detect Prev, 29 (2005), 544-551.

[18] A. A. Khalil, N. F. Kabapy, S. F. Deraz, and C. Smith, Heat shock proteins in oncology: diagnostic biomarkers or therapeutic targets?, Biochim Biophys Acta, 1816 (2011), 89-104.

[19] D. R. Ciocca and S. K. Calderwood, Heat shock proteins in cancer: diagnostic, prognostic, predictive, and treatment implications, Cell Stress Chaperones, 10 (2005), 86-103.

[20] S. E. Conroy, S. L. Gibson, G. Brunstrom, D. Isenberg, Y. Luqmani, and D. S. Latchman, Autoantibodies to $90 \mathrm{kD}$ heat-shock protein in sera of breast cancer patients, Lancet, 345 (1995), 126.

[21] S. E. Conroy, P. D. Sasieni, I. Fentiman, and D. S. Latchman, Autoantibodies to the 90kDa heat shock protein and poor survival in breast cancer patients, Eur J Cancer, 34 (1998), 942-943.

[22] S. E. Conroy, P. D. Sasieni, V. Amin, D. Y. Wang, P. Smith, I. S. Fentiman, et al., Antibodies to heatshock protein 27 are associated with improved survival in patients with breast cancer, Br J Cancer, 77 (1998), 1875-1879.

[23] M. A. Fanelli, F. D. Cuello Carrion, J. Dekker, J. Schoemaker, and D. R. Ciocca, Serological detection of heat shock protein hsp27 in normal and breast cancer patients, Cancer Epidemiol Biomarkers Prev, 7 (1998), 791-795.

[24] B. Hamrita, K. Chahed, M. Kabbage, C. L. Guillier, M. Trimeche, A. Chaieb, et al., Identification of tumor antigens that elicit a humoral immune response in breast cancer patients' sera by serological proteome analysis (SERPA), Clin Chim Acta, 393 (2008), 95-102.

[25] C. Desmetz, F. Bibeau, F. Boissiere, V. Bellet, P. Rouanet, T. Maudelonde, et al., Proteomics-based identification of HSP60 as a tumor-associated antigen in early stage breast cancer and ductal carcinoma in situ, J Proteome Res, 7 (2008), 3830-3837.

[26] L. Y. Luo, I. Herrera, A. Soosaipillai, and E. P. Diamandis, Identification of heat shock protein 90 and other proteins as tumour antigens by serological screening of an ovarian carcinoma expression library, Br J Cancer, 87 (2002), 339-343.

[27] I. Korneeva, A. M. Bongiovanni, M. Girotra, T. A. Caputo, and S. S. Witkin, Serum antibodies to the 27-kd heat shock protein in women with gynecologic cancers, Am J Obstet Gynecol, 183 (2000), 18-21. 
[28] C. I. Vidal, P. J. Mintz, K. Lu, L. M. Ellis, L. Manenti, R. Giavazzi, et al., An HSP90-mimic peptide revealed by fingerprinting the pool of antibodies from ovarian cancer patients, Oncogene, 23 (2004), 8859-8867.

[29] K. S. Looi, E. S. Nakayasu, R. A. Diaz, E. M. Tan, I. C. Almeida, and J. Y. Zhang, Using proteomic approach to identify tumor-associated antigens as markers in hepatocellular carcinoma, J Proteome Res, 7 (2008), 4004-4012.

[30] Q. Shao, P. Ren, Y. Li, B. Peng, L. Dai, N. Lei, et al., Autoantibodies against glucose-regulated protein 78 as serological diagnostic biomarkers in hepatocellular carcinoma, Int J Oncol, 41 (2012), 1061-1067.

[31] M. Takashima, Y. Kuramitsu, Y. Yokoyama, N. Iizuka, T. Harada, M. Fujimoto, et al., Proteomic analysis of autoantibodies in patients with hepatocellular carcinoma, Proteomics, 6 (2006), 3894-3900.

[32] Y. Fujita, T. Nakanishi, Y. Miyamoto, M. Hiramatsu, H. Mabuchi, A. Miyamoto, et al., Proteomicsbased identification of autoantibody against heat shock protein 70 as a diagnostic marker in esophageal squamous cell carcinoma, Cancer Lett, 263 (2008), 280-290.

[33] J. Zhang, K. Wang, J. Zhang, S. S. Liu, L. Dai, and J. Y. Zhang, Using proteomic approach to identify tumor-associated proteins as biomarkers in human esophageal squamous cell carcinoma, J Proteome Res, 10 (2011), 2863-2872.

[34] S. Tsunemi, T. Nakanishi, Y. Fujita, G. Bouras, Y. Miyamoto, A. Miyamoto, et al., Proteomics-based identification of a tumor-associated antigen and its corresponding autoantibody in gastric cancer, Oncol Rep, 23 (2010), 949-956.

[35] Y. He, Y. Wu, Z. Mou, W. Li, L. Zou, T. Fu, et al., Proteomics-based identification of HSP60 as a tumor-associated antigen in colorectal cancer, Proteomics Clin Appl, 1 (2007), 336-342.

[36] P. J. Mintz, J. Kim, K. A. Do, X. Wang, R. G. Zinner, M. Cristofanilli, et al., Fingerprinting the circulating repertoire of antibodies from cancer patients, Nat Biotechnol, 21 (2003), 57-63.

[37] L. Zhong, X. Peng, G. E. Hidalgo, D. E. Doherty, A. J. Stromberg, and E. A. Hirschowitz, Antibodies to HSP70 and HSP90 in serum in non-small cell lung cancer patients, Cancer Detect Prev, 27 (2003), 285-290.

[38] J. Fan, S. Upadhye, and A. Worster, Understanding receiver operating characteristic (ROC) curves, Cjem, 8 (2006), 19-20.

[39] C. Desmetz, C. Bascoul-Mollevi, P. Rochaix, P. J. Lamy, A. Kramar, P. Rouanet, et al., Identification of a new panel of serum autoantibodies associated with the presence of in situ carcinoma of the breast in younger women, Clin Cancer Res, 15 (2009), 4733-4741.

[40] Z. Yang, Y. Chevolot, T. Gehin, J. Solassol, A. Mange, E. Souteyrand, et al., Improvement of protein immobilization for the elaboration of tumor-associated antigen microarrays: application to the sensitive and specific detection of tumor markers from breast cancer sera, Biosens Bioelectron, 40 (2012), 385392.

[41] H. Liu, J. Zhang, S. Wang, Z. Pang, Z. Wang, W. Zhou, et al., Screening of autoantibodies as potential biomarkers for hepatocellular carcinoma by using T7 phase display system, Cancer Epidemiol, 36 (2012), 82-88.

[42] C. H. Middleton, W. Irving, J. F. Robertson, A. Murray, C. B. Parsy-Kowalska, I. K. Macdonald, et al., Serum autoantibody measurement for the detection of hepatocellular carcinoma, PLoS One, 9 (2014), 19.

[43] P. Massoner, A. Lueking, H. Goehler, A. Hopfner, A. Kowald, K. G. Kugler, et al., Serumautoantibodies for discovery of prostate cancer specific biomarkers, Prostate, 72 (2012), 427-436.

[44] L. Zhong, X. Peng, G. E. Hidalgo, D. E. Doherty, A. J. Stromberg, and E. A. Hirschowitz, Identification of circulating antibodies to tumor-associated proteins for combined use as markers of non-small cell lung cancer, Proteomics, 4 (2004), 1216-1225.

[45] E. C. Farlow, K. Patel, S. Basu, B. S. Lee, A. W. Kim, J. S. Coon, et al., Development of a multiplexed tumor-associated autoantibody-based blood test for the detection of non-small cell lung cancer, Clin Cancer Res, 16 (2010), 3452-3462.

[46] F. J. Lowe, W. Shen, J. Zu, J. Li, H. Wang, X. Zhang, et al., A novel autoantibody test for the detection of pre-neoplastic lung lesions, Mol Cancer, 13 (2014), 1-12.

[47] R. Rucksaken, C. Pairojkul, P. Pinlaor, N. Khuntikeo, S. Roytrakul, C. Selmi, et al., Plasma Autoantibodies against Heat Shock Protein 70, Enolase 1 and Ribonuclease/Angiogenin Inhibitor 1 as Potential Biomarkers for Cholangiocarcinoma, PLoS One, 9 (2014), 1-12.

[48] B. M. Nolen and A. E. Lokshin, Autoantibodies for cancer detection: still cause for excitement?, Cancer Biomark, 6 (2010), 229-245.

[49] M. Karlsson, I. Mollegard, G. Stiernstedt, and B. Wretlind, Comparison of Western blot and enzymelinked immunosorbent assay for diagnosis of Lyme borreliosis, Eur J Clin Microbiol Infect Dis, 8 (1989), 871-877. 
[50] F. X. Sutandy, J. Qian, C. S. Chen, and H. Zhu, Overview of protein microarrays, Curr Protoc Protein Sci, 27 (2013), 1-21.

Table 1 Frequency of single anti-HSP autoantibody in cancer patients (cases), benign subjects and healthy controls (HC).

\begin{tabular}{|c|c|c|c|c|c|c|c|c|c|c|}
\hline \multirow{2}{*}{ cancer } & \multirow{2}{*}{ method } & \multirow{2}{*}{$\begin{array}{c}\text { Anti-HSP } \\
\text { detected }\end{array}$} & \multicolumn{3}{|c|}{ sample size $(\mathrm{N})$} & \multicolumn{3}{|c|}{$\mathrm{AAb}$ frequency $\%$} & \multirow{2}{*}{$P$ value } & \multirow{2}{*}{ reference } \\
\hline & & & cases & $\mathrm{HC}$ & benign & cases & $\mathrm{HC}$ & benign & & \\
\hline \multirow{7}{*}{ Breast } & ELISA & Anti- HSPB1 & 579 & 53 & - & $37.8 \%$ & $1.9 \%$ & - & $\mathrm{p}<0.001$ & [22] \\
\hline & Immunoblot & Anti-HSPB1 & 11 & 5 & - & 0 & 0 & - & - & [23] \\
\hline & ELISA & Anti-HSP70 & 369 & 53 & - & $40.9 \%$ & $35.9 \%$ & - & - & [22] \\
\hline & ELISA & Anti-HSP90 & 125 & - & - & $36.8 \%$ & - & - & - & [20] \\
\hline & Immunoblot & Anti-HSPD1 & 40 & 42 & - & $47.5 \%$ & $4.7 \%$ & - & $\mathrm{p}<0.01$ & [24] \\
\hline & ELISA & Anti-HSPD1 & 107 & 93 & - & $31.8 \%$ & $4.3 \%$ & - & $\mathrm{p}<0.0001$ & [25] \\
\hline & ELISA & Anti-HSP90 & 13 & 22 & 10 & $8 \%$ & 0 & 0 & - & [26] \\
\hline \multirow[t]{7}{*}{ Ovarian } & ELISA & Anti-HSPB1 & 34 & 29 & 23 & $50 \%$ & $3.4 \%$ & $4.3 \%$ & $\mathrm{p}=0.0003$ & [27] \\
\hline & ELISA & Anti-HSPD1 & 31 & 29 & 22 & $19.4 \%$ & $13.8 \%$ & $18.2 \%$ & - & [27] \\
\hline & ELISA & Anti-HSP70 & 30 & 29 & 23 & $13.3 \%$ & $24.1 \%$ & $13 \%$ & - & [27] \\
\hline & ELISA & Anti-HSP90 & 32 & 29 & 23 & $8.8 \%$ & $6.9 \%$ & $13 \%$ & - & [27] \\
\hline & ELISA & Anti-HSPD1 & 10 & 93 & - & 0 & 0 & - & - & [25] \\
\hline & ELISA & Anti-HSP90 & 32 & 22 & 20 & $25 \%$ & 0 & $5 \%$ & - & [26] \\
\hline & ELISA & $\begin{array}{l}\text { Anti-HSP90- } \\
\text { mimic } \\
\text { peptide }\end{array}$ & $59^{*}$ & 57 & 30 & $22 \%$ & $14 \%$ & $3.3 \%$ & - & [28] \\
\hline \multirow[t]{4}{*}{$\mathrm{HCC}$} & ELISA & Anti-HSPD1 & 20 & 24 & 60 & $20 \%$ & $3.3 \%$ & $31.7 \%$ & - & [29] \\
\hline & ELISA & Anti-HSP70 & 20 & 24 & 60 & $25 \%$ & 0 & $1.6 \%$ & $\mathrm{p}<0.01$ & [29] \\
\hline & ELISA & Anti-HSPA5 & 76 & 86 & 60 & $35.5 \%$ & 0 & 0 & $\mathrm{p}<0.01$ & [30] \\
\hline & Immunoblot & Anti-HSP70 & 15 & 20 & - & $46.7 \%$ & $10 \%$ & - & $\mathrm{p}=0.014$ & [31] \\
\hline \multirow{3}{*}{ ESCC } & ELISA & Anti-HSP70 & 16 & 13 & - & $93.7 \%$ & 0 & - & $p<0.001$ & [32] \\
\hline & ELISA & Anti-HSP70 & 69 & 76 & - & $39.1 \%$ & $1.3 \%$ & - & $\mathrm{p}<0.01$ & [33] \\
\hline & Immunoblot & Anti-HSPA5 & 15 & 20 & - & $26.7 \%$ & 0 & - & - & [34] \\
\hline \multirow[t]{4}{*}{ CRC } & ELISA & Anti-HSP90 & 37 & 22 & - & $3 \%$ & 0 & - & - & [26] \\
\hline & ELISA & Anti-HSPD1 & 60 & 30 & - & - & - & - & $\mathrm{p}<0.01$ & [35] \\
\hline & ELISA & Anti-HSP70 & 19 & 13 & - & $21 \%$ & 0 & - & - & [32] \\
\hline & Immunoblot & Anti-HSPA5 & 15 & 20 & - & $20 \%$ & 0 & - & - & [34] \\
\hline \multirow[t]{2}{*}{ Gastric } & ELISA & Anti-HSP70 & 17 & 13 & - & $11.7 \%$ & 0 & - & - & [32] \\
\hline & Immunoblot & Anti-HSPA5 & 60 & 20 & - & $28.3 \%$ & 0 & - & - & [34] \\
\hline \multirow[t]{3}{*}{ Prostate } & ELISA & Anti-HSP90 & 20 & 22 & - & 0 & 0 & - & - & [26] \\
\hline & ELISA & Anti-HSPA5 & 108 & 71 & - & $37 \%$ & $7 \%$ & - & - & [36] \\
\hline & ELISA & $\begin{array}{l}\text {-peptide } \\
\text { Anti-HSPD1 }\end{array}$ & 10 & 93 & - & 0 & 0 & - & - & [25] \\
\hline \multirow[t]{3}{*}{ NSCLC } & ELISA & Anti-HSP90 & 10 & 22 & - & 0 & 0 & - & - & [26] \\
\hline & ELISA & Anti-HSP70 & 49 & 40 & - & $10.2 \%$ & - & - & - & [37] \\
\hline & ELISA & Anti-HSP90 & 49 & 40 & - & - & - & - & $\mathrm{P}=0.11$ & [37] \\
\hline
\end{tabular}

$H C$ healthy controls, HCC hepatocellular carcinoma, ESCC Esophageal squamous cell carcinoma, CRC colorectal cancer, NSCLC non-small cell lung cancer, ELISA enzyme linked immunosorbent assay

${ }^{*}$ Samples from ascites, otherwise from sera 
Table 2 Frequency of AAbs panels in cancers and controls

\begin{tabular}{|c|c|c|c|c|c|c|}
\hline Cancer & methods & Autoantibody panel & cases & controls & SN/SP/AUC & reference \\
\hline \multirow[t]{2}{*}{ Breast } & ELISA & $\begin{array}{l}\text { Anti-FKBP52, anti-PPIA, } \\
\text { anti-PRDX2, anti-HSPD1 } \\
\text { and anti-MUC1 }\end{array}$ & 142 & 93 & $60.5 \% / 77.2 \% / 0.74$ & [39] \\
\hline & PM & $\begin{array}{l}\text { Anti-HSPD1, anti-P53, } \\
\text { anti-Her2, anti-NY-ESO-1 } \\
\text { and anti-HSP70 }\end{array}$ & 29 & 28 & $82.7 \% /-/-$ & [40] \\
\hline \multirow[t]{3}{*}{$\mathrm{HCC}$} & ELISA & $\begin{array}{l}\text { Anti-CENPF, anti-DDX3, } \\
\text { anti-HSP70, anti-HSPA5, } \\
\text { anti-VIM, anti-LMNB1 } \\
\text { and anti-p53 }\end{array}$ & 70 & 120 & $82.86 \% / 100 \% / 0.92$ & [41] \\
\hline & ELISA & Anti-HSPA5, anti-AFP & 63 & 86 & $71.4 \% /-/-$ & [30] \\
\hline & ELISA & $\begin{array}{l}\text { Anti-AFP, anti-Cyclin B1, } \\
\text { anti-Gankyrin, anti-P53, } \\
\text { anti-NY-ESO-1, anti-RalA, } \\
\text { anti-CK8, anti-HSPA5, } \\
\text { anti-HDGF and anti-DKK1 }\end{array}$ & 96 & 96 & $41 \% / 91 \% /-$ & [42] \\
\hline \multirow[t]{2}{*}{ ESCC } & ELISA & $\begin{array}{l}\text { Anti-P53, anti-NY-ESO-1, } \\
\text { anti-MMP-7, anti-HSP70, } \\
\text { anti-Prx VI and anti-Bmi-1 }\end{array}$ & 388 & 125 & $45 \% / 95 \% /-$ & [10] \\
\hline & ELISA & $\begin{array}{l}\text { Anti-P53, anti-NY-ESO-1, } \\
\text { anti-MMP-7, anti-HSP70, } \\
\text { anti-Prx VI and anti-Bmi-1 }\end{array}$ & 237 & 134 & $46 \% / 96 \% /-$ & [10] \\
\hline Prostate & PM & $\begin{array}{l}\text { Anti-TTLL12, anti- } \\
\text { VAT1L, anti-GGA1, anti- } \\
\text { RPIA, anti-NHSL1, anti- } \\
\text { NOVA2, anti-MAP2, anti- } \\
\text { PRPF38B, anti-SRP14, } \\
\text { anti-HSP110, anti- } \\
\text { ZNF154, anti-ADD1, anti- } \\
\text { RASSF7 and anti-RBM15 }\end{array}$ & 40 & 40 & $-/-/ 0.69$ & [43] \\
\hline \multirow[t]{4}{*}{ NSCLC } & ELISA & Anti-HSP70, anti-HSP90 & 49 & 40 & $78 \% / 65 \% / 0.74$ & [37] \\
\hline & ELISA & $\begin{array}{l}\text { Anti-HSP70, anti-HSP90, } \\
\text { anti-p130, anti-GAGE, and } \\
\text { anti-BMI-1 }\end{array}$ & 16 & 40 & $82 \% / 83 \% / 0.84$ & [44] \\
\hline & $\begin{array}{l}\text { Immunobead } \\
\text { assay }\end{array}$ & $\begin{array}{l}\text { Anti-phosphoglycerate } \\
\text { mutase, anti-ubiquillin, } \\
\text { anti-Annexin I, anti- } \\
\text { Annexin II, anti-IMPDH } \\
\text { and anti-HSP70 }\end{array}$ & 117 & 79 & $-/-/ 0.93$ & [45] \\
\hline & PM & $\begin{array}{l}\text { Anti-HSP70, anti-LTBP1, } \\
\text { anti-BMI1, anti-GAGE7, } \\
\text { anti-AGBL5, anti-HES1, } \\
\text { anti-PDE4A, anti-NEFH, } \\
\text { and anti-cDNA FLJ45990 }\end{array}$ & 100 & 200 & $82 \% / 70 \% / 0.81$ & [46] \\
\hline $\mathrm{CCA}$ & ELISA & $\begin{array}{l}\text { Anti-HSP70, anti-ENO1 } \\
\text { and anti-RNH1 }\end{array}$ & 31 & 23 & $71 \% / 83 \% /-$ & [47] \\
\hline
\end{tabular}

$H C$ healthy controls, $H C C$ hepatocellular carcinoma, ESCC Esophageal squamous cell carcinoma, CRC colorectal cancer, NSCLC non-small cell lung cancer, CCA cholangiocarcinoma, ELISA enzyme linked immunosorbent assay, $P M$ protein microarray, $S N$ sensitivity, $S P$ specificity, $A U C$ Area under curve, FKBP52 FK506 binding proteins, PPIA Peptidylprolyl Isomerase A, $P R D X 2$ peroxiredoxin 2, MUC1 mucin glycoproteins family, Her2-Fc human epidermal factor receptor, $N Y$-ESO-1 Cancer/testis antigen 1, CENPF centromere protein F, DDX3 DEAD (Asp-Glu-Ala-Asp) box helicase 3, VIM vimentin, LMNB1 lamin B1, AFP alpha-fetoprotein, RalA Ras-related protein, $C K 8$ Cytokeratin 8, HDGF hepatoma-derived growth factor, $D K K 1$ dickkopf WNT signaling pathway inhibitor 1, MMP-7 matrix metallopeptidase 7, Prx VI peroxiredoxin 6, Bmi-1 Polycomb 
complex protein, TTLL12 Tubulin-tyrosine ligase-like protein 12, VAT1L vesicle amine transport protein 1, GGAl golgi-associated, gamma adaptin ear containing, ARF binding protein 1, RPIA ribose 5-phosphate isomerase A, NHSL1 NHS-like protein 1, NOVA2 neuro-oncological ventral antigen 2, MAP2 Microtubuleassociated protein 2, PRPF38B pre-mRNA processing factor 38B, SRP14 signal recognition particle $14 \mathrm{kDa}$, ZNF154 zinc finger protein 154, ADD1 adducin 1, RASSF7 Ras association (RalGDS/AF-6) domain family (Nterminal) member 7, RBM15 RNA binding motif protein 15, GAGE G antigen, IMPDH Inosine monophosphate dehydrogenase, LTBPl latent transforming growth factor beta binding protein, AGBL5 ATP/GTP binding protein-like 5, HES1 Transcription factor, PDE4A phosphodiesterase 4A, NEFH neurofilament, heavy polypeptide, cDNA FLJ45990 zinc finger and BTB domain containing 7B, ENO1 enolase 1, RNH1 ribonuclease/angiogenin inhibitor 1

Table 3: Comparison of anti-HSP AAbs frequencies in various cancers.

\begin{tabular}{|c|c|c|c|c|c|c|c|c|c|}
\hline \multirow{2}{*}{$\begin{array}{l}\text { Anti-HSP } \\
\mathrm{AAb}\end{array}$} & \multirow[t]{2}{*}{ Cancer } & \multirow[t]{2}{*}{ Method } & \multicolumn{3}{|c|}{ sample size $(\mathrm{N})$} & \multicolumn{3}{|c|}{$\mathrm{AAb}$ frequency $\%$} & \multirow[t]{2}{*}{ Reference } \\
\hline & & & cases & $\mathrm{HC}$ & benign & cases & $\mathrm{HC}$ & benign & \\
\hline Anti- & Breast & ELISA & 579 & 53 & - & $37.8 \%$ & $1.9 \%$ & - & {$[22]$} \\
\hline \multirow[t]{2}{*}{ HSPB 1} & Breast & Immunoblot & 11 & 5 & - & 0 & 0 & - & [23] \\
\hline & Ovarian & ELISA & 34 & 29 & 23 & $50 \%$ & $3.4 \%$ & $4.3 \%$ & [27] \\
\hline \multirow{7}{*}{$\begin{array}{l}\text { Anti- } \\
\text { HSPD1 }\end{array}$} & Breast & Immunoblot & 40 & 42 & - & $47.5 \%$ & $4.7 \%$ & - & [24] \\
\hline & Breast & ELISA & 107 & 93 & - & $31.8 \%$ & $4.3 \%$ & - & [25] \\
\hline & Ovarian & ELISA & 31 & 29 & 22 & $19.4 \%$ & $13.8 \%$ & $18.2 \%$ & [27] \\
\hline & Ovarian & ELISA & 10 & 93 & - & 0 & 0 & - & {$[25]$} \\
\hline & $\mathrm{HCC}$ & ELISA & 20 & 24 & 60 & $20 \%$ & $3.3 \%$ & $31.7 \%$ & [29] \\
\hline & $\mathrm{HCC}$ & ELISA & 70 & 70 & 50 & $20 \%$ & $1.43 \%$ & $6 \%$ & [41] \\
\hline & Prostate & ELISA & 10 & 93 & - & 0 & 0 & - & [25] \\
\hline \multirow{12}{*}{$\begin{array}{l}\text { Anti- } \\
\text { HSP70 }\end{array}$} & Breast & ELISA & 369 & 53 & - & $40.9 \%$ & $35.9 \%$ & - & [22] \\
\hline & Ovarian & ELISA & 30 & 29 & 23 & $13.3 \%$ & $24.1 \%$ & $13 \%$ & [27] \\
\hline & $\mathrm{HCC}$ & ELISA & 20 & 24 & 60 & $25 \%$ & 0 & $1.6 \%$ & [29] \\
\hline & $\mathrm{HCC}$ & Immunoblot & 15 & 20 & - & $46.7 \%$ & $10 \%$ & - & [31] \\
\hline & $\mathrm{HCC}$ & ELISA & 70 & 70 & 50 & $37.15 \%$ & 0 & 0 & [41] \\
\hline & ESCC & ELISA & 16 & 13 & - & $93.7 \%$ & 0 & - & [32] \\
\hline & ESCC & ELISA & 69 & 76 & - & $39.1 \%$ & $1.3 \%$ & - & [33] \\
\hline & ESCC & ELISA & 388 & 125 & - & $11 \%$ & - & - & [10] \\
\hline & ESCC & ELISA & 237 & 134 & - & $8 \%$ & - & - & [10] \\
\hline & $\mathrm{CRC}$ & ELISA & 19 & 13 & - & $21 \%$ & 0 & - & [32] \\
\hline & Gastric & ELISA & 17 & 13 & - & $11.7 \%$ & 0 & - & [32] \\
\hline & NSCLC & ELISA & 49 & 40 & - & $10.2 \%$ & - & - & [37] \\
\hline \multirow{7}{*}{$\begin{array}{l}\text { Anti- } \\
\text { HSPA5 }\end{array}$} & $\mathrm{HCC}$ & ELISA & 76 & 86 & 60 & $35.5 \%$ & 0 & 0 & [30] \\
\hline & $\mathrm{HCC}$ & ELISA & 70 & 70 & 50 & $27.14 \%$ & 0 & 0 & [41] \\
\hline & $\mathrm{HCC}$ & ELISA & 96 & 96 & - & $6 \%$ & - & - & [42] \\
\hline & ESCC & Immunoblot & 15 & 20 & - & $26.7 \%$ & 0 & - & [34] \\
\hline & $\mathrm{CRC}$ & Immunoblot & 15 & 20 & - & $20 \%$ & 0 & - & [34] \\
\hline & Gastric & Immunoblot & 60 & 20 & - & $28.3 \%$ & 0 & - & [34] \\
\hline & Prostate & ELISA & 108 & 71 & - & $37 \%$ & $7 \%$ & - & [36] \\
\hline \multirow{9}{*}{$\begin{array}{l}\text { Anti- } \\
\text { HSP90 }\end{array}$} & Breast & ELISA & 125 & - & - & $36.8 \%$ & - & - & [20] \\
\hline & Breast & ELISA & 13 & 22 & 10 & $8 \%$ & 0 & 0 & [26] \\
\hline & Ovarian & ELISA & 32 & 29 & 23 & $8.8 \%$ & $6.9 \%$ & $13 \%$ & [27] \\
\hline & Ovarian & ELISA & 32 & 22 & 20 & $25 \%$ & 0 & $5 \%$ & [26] \\
\hline & Ovarian & ELISA & $59^{*}$ & 57 & 30 & $22 \%$ & $14 \%$ & $3.3 \%$ & [28] \\
\hline & $\mathrm{HCC}$ & ELISA & 70 & 70 & 50 & $8.57 \%$ & $1.43 \%$ & $2 \%$ & [41] \\
\hline & $\mathrm{CRC}$ & ELISA & 37 & 22 & - & $3 \%$ & 0 & - & [26] \\
\hline & Prostate & ELISA & 20 & 22 & - & 0 & 0 & - & [26] \\
\hline & NSCLC & ELISA & 10 & 22 & - & 0 & 0 & - & [26] \\
\hline
\end{tabular}

HC healthy controls, $H C C$ hepatocellular carcinoma, ESCC Esophageal squamous cell carcinoma, $C R C$ colorectal cancer, NSCLC non-small cell lung cancer, CCA cholangiocarcinoma, ELISA enzyme linked immunosorbent assay

*Samples from ascites, otherwise from sera 